\title{
RESULTADO DO ESTUDO DE UM PEQUENO LOTE DE OPILIŐES
}

\author{
S. de Toledo Piza Júnior \\ Professor de Zoologia, Anatomia e Fisiologia \\ da Escola Superior de Agricultura "Luiz de Queiroz" \\ da Universidade de Săo Paulo
}

\section{INDICE}




\section{GONYLEPTIDAE-PACHYLINAE}

Eusarcus sulcatus (Piza, 1940)

Goyazia sulcata Piza, 1940 : Arq. Zool. S. Paulo, Vol. I, Pag. 54, Fig. 2.

Eusarcus sulcatus, Soares : 1946, Arq. Zool. S. Paulo, Vol. IV, Pag. 519.

Esta espécie foi descrita com base num macho apanhado em Anápolis, E. de Goiás, pelo Dr. Frederico Lane e referido como fêmea na descrição original. MELO-LEITAOO (1945 - An. Acad. Bras. Cien. XIV (2) : 156), embora devesse ter visto pela descrição do tipo tratar-se de macho, acha que a espécie deve ser sinônima de Eusarcus bifidus Rwr., 1929, da qual representaria a fêmea. SOARES (1946 - Papéis Avulsos do Dep. Zool. S. Paulo, Vol. VII, N. ${ }^{\circ}$ 20, Pag. 237) descreveu a fếmea de Eusarcus bifidus, tirando com isso a dúvida posta por MELOLEITAO sôbre a validade da minha espécle.

Havendo recebido do Estado de Mato Grosso machos e fêmeas de Eusarcus sulcatus, verifico tratar-se realmente de uma boa espécie, aproveitando, porisso, esta oportunidade, para redescrevé-la :

Mas : $4 \mathrm{~mm}$. Articuli tarsales : $6-$ plus q. $6-6-6$.

Margo anterior cephalothoracis rectus, superne ad latera tantum granulatus seu laevis, antice dentibus tribus armatus. Cephalothorax plus minusve distincte parce granulatus. Tuber oculorum ante medium cephalothoracis, magnum, elevatum, in apophyse bifida terminatum, granulis nonnullis postice praeditum. Scutum dorsale minute granulatum, granulis arearum II-IV plus minusve distincte bi-seriatim ordinatis. Area I in medio longitudinaliter divisa. Area III spina adunca in medio armata. Area V ac segmenta abdominalia dorsalia libera ordine granulorum. Areae laterales granulatae, ad margines ordine granulorum magnorum sensim prominentiorum, quorum ultimum dentiforme intructae. Operculum anale dorsale fortiter granulatum. Coxae omnes ac area spiraculorum densc granulatae. Segmenta abdominalia ventralia ordine granulorum. Femur palporum superne ordine granulorum magnorum, intus ad extremitatem spina apicali, inferne spina basali forti armatum. Femora pedum I et II recta, III modice arquata, IV fortiter sinuosa, omnia granulata. Pedes III : trochanteres granulati, spina apicali parva intus armati; femora inferne 
ad dmidiam partem apicalem dentata, apice superne dentibus fortioribus armato; patellae crassae, granulatae; tibiae fortes, femoribus crassiores, inferne bi-seriatim dentatae. Pede: IV : coxae prominenter granulatae, apophyse apicali externa crassa, deflexa, obtusa, instructae; trochanteres granulati, extus ad basin processu forti, humili, intus, ad apicem apophyse dentiformi fragili praediti; femora et patellae et tibiae ut in pedibus II sed paullo crassiora.

Castaneus uniformis.

Femina (Allotipus) (Fig. 1) Maris simillis sed pedibus III el IV gracilioribus, coxis IV apophyse apicali externa spiniformi parvulaque, trochanteribus apophyse apicali interna parvulissima tantum, tibiis III femoribus haud crassioribus.

Habitat: Jupiá, Três Lagoas (E. de Mato Grosso).

Col.: A. Zamith \& A. Corrêa, XII-1946.

5 machos e 7 fêmeas (alótipos) no Laboratório do autor.

Yraguara annulipes M.-L. 1936

Yraguara annulipes Melo-Leitão, 1935-1936 : Mem. Ins. Butatan, Vol. X, Pag. 291, Fig. 2.

Melloleitanius barretensis Piza, 1938 : Folia Clin. et Biol. Vol. X (4), Pag. 115, Fig. 2.

Yraguara barretensis, Soares, 1943 : Papéis Avulsos Dep. Zool. S. Paulo, Vol. III (13), Pag. 210.

Pelo estudo de dois machos e uma fêmea coligidos por A. Zamith \& A. Corrêa em Avanhandava no mês de Dezembro de 1946 e três machos e três fêmeas coligidos em Penápolis na mesma ocasião e pelas mesmas pessóas, conclú que Yraguara barretensis (Piza) é sinônima de Yraguara annulipes Melo Leitão.

Sirvo-me desta oportunidade para dar a descriçăo do alótipo.

Femina (Fig. 2) : Margo anterior cephalothoracis inermis. Cephalothorax granulatus. Tuber oculorum prope marginem cephalothoracis anteriorem situm, elevatum, granulatum, sp'nis dentiformibus conicis appropinquatis duabus armatum. Scutum abdominale granulatum, areis I et IV in medio longitudinaliter divisis, III et IV tuberculis parvulis acutis duobuis in medio praeditis. Area $\mathrm{V}$ ac segmenta abdominalia dorsalia libera ordinibus granulorum duobus necnon granulis nonnullis prominentioribus armata. Operculum anale dorsale granulatum. Areae laterales ordinibus granulorum duobus, granulis ordinis externis quam internis majoribus. Segmenta ventralia libera ac operculum anale ventrale humiliter granulata. Femora III et 
IV arquata. Femora palporum intus ad apicem inermia. Pedes. IV : coxae dense granulatae, spina apicali parva, dentiformi, armatae; femora acute granulata, ad apicem dentada; patellae tibiaeque acute granulatae.

Articulis tarsales : $6-7-6-6$.

Longitudo : $6 \mathrm{~mm}$.

Castanea, femoribus tibilsque plus minusve distincte late nigro-annulatis.

Habitat : Penápolis, E. de S. Paulo.

Col.: A. Zamith \& A. Corrêa XII-1946.

Alótipos : 4 fêmeas no Laboratório do autor.

\section{COSMETIDAE - COSMETINAE}

Elleria gen. nov.

Articuli tarsales : 5 - plus q. $6-5-6$.

Area I scuti dorsalis tuberculis prominentibus duobus, area III processibus conicis prominentioribus, area IV spinis conicis crassisque duabus in medio armatae. Area II ac $V$ inermes. Segmenta abdominalia dorsalia libera atque operculum anale dorsali inermia. Pedes graciles.

O presente gênero, cujo nome é dado em homenagem á Exma. Sra. D. Elia Heller Monteiro Soares, que também se dedica ao estudo dos Opiliões, é baseado numa única fêmea, que constitui o tipo da espécie seguinte.

Elleria bilunata sp. n. (Fig. 3)

Femina : $4 \mathrm{~mm}$. Articuli tarsales : $5-$ plus q. $6-5-6$.

Corpus castaneo-nigrum, granulis prominentibus albis omnino obtectum. Area I tuberculis prominentibus duobus in medio necnon maculis magnis lunatis, albis, duabus, sulco II tangentibus praedita. Area II inermis. Area III processibus conicis duobus tuberculis areae I prominentioribus in medio armata. Area IV spnis conicis duabus in medio instructa. Area $V$ ac segmenta abdominalia dorsalia libera ordine granulorum. Operculum anale dorsale nigrum, grosse granulatum. Coxae I distincte, II et III obsolete granulatae. Area stigmatica haud granulata sed opaca. Segmenta abdominalia ventralia ordine granulorum minutissimorum. Pedes graciles, femoribus III-IV modice sinuosis. Coxae IV lateraliter grosse granulatae, antice ad basin tuberculo transverso, ad apicem dente crasso extus armatae.

Pátria : Jupiá, Três Lagoas (E. de Mato Grosso).

Col. : A. Zamith \& A. Corrêa, XII-1946.

Tipo : Uma fêmea, no Laboratório do A. 


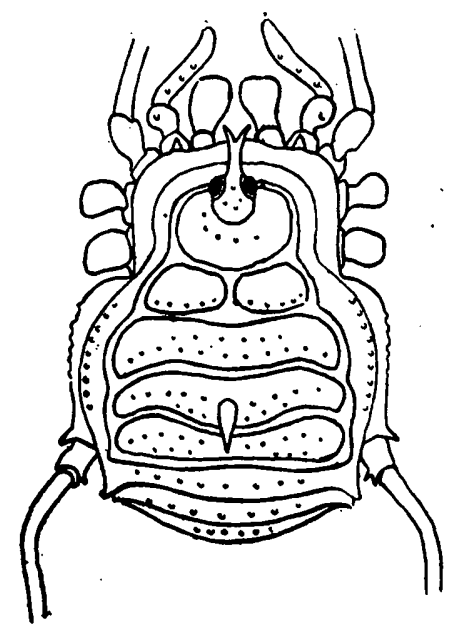

Fig. 1 - Eusarcus sulcatus (Piza) (alótipo fêmea)

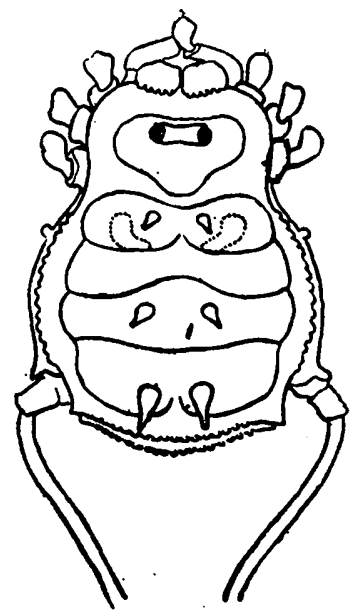

Fig. 3 - Elleria bilunata sp. n. (fêmea)

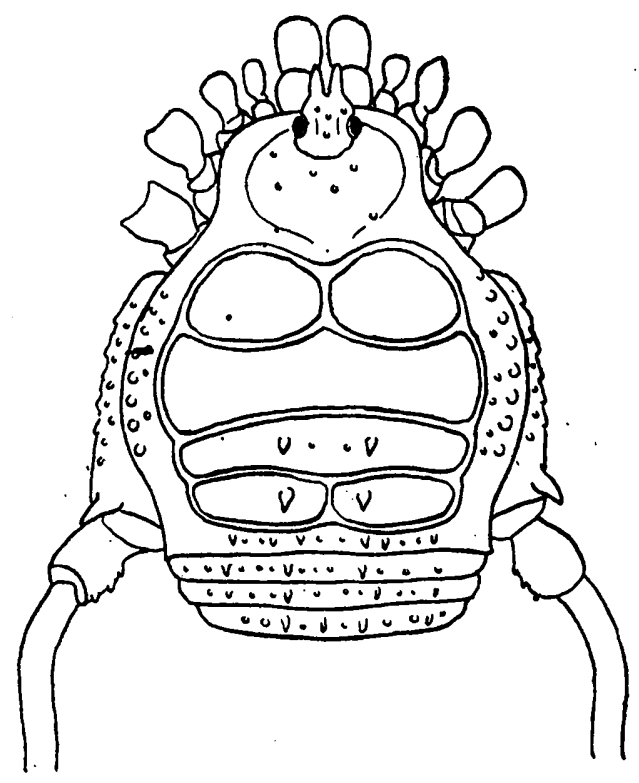

Fig. 2 - Yraguara annulipes M. L. (alótipo fêmea) 